\title{
Pre-chemotherapy preservation of fertility in male patients with high-grade malignant bone and soft tissue tumors
}

\author{
MANABU HOSHI $^{1}$, NAOTO OEBISU ${ }^{1}$, JUN TAKADA ${ }^{1}$, TADASHI IWAI ${ }^{1}$, \\ RIE TSURUTA $^{2}$ and HIROAKI NAKAMURA ${ }^{1}$ \\ Departments of ${ }^{1}$ Orthopedic Surgery and ${ }^{2}$ Nursing Office, Osaka City University \\ Graduate School of Medicine, Osaka 545-8585, Japan
}

Received June 24, 2014; Accepted July 21, 2014

DOI: $10.3892 /$ mco. 2014.367

\begin{abstract}
Only a limited number of orthopedic oncologists make arrangements for pre-chemotherapy fertility preservation, such as sperm cryopreservation, for their patients. The purpose of this study was to offer fertility preservation to male patients with high-grade malignant bone and soft tissue tumors and assess the outcomes. The study included 14 male patients, aged $<45$ years, with high-grade bone and soft tissue tumors. The median age at diagnosis was $23.0 \pm 12.0$ years (range, 8-42 years). Following pathological confirmation of high-grade malignant tumor, we informed all the patients and/or their guardians on the issue of chemotherapy-related male infertility. If the patients were interested in preserving fertility, they were referred to a team of reproductive specialists. We documented the patients' clinical characteristics and techniques used for fertility preservation. The majority of unmarried and childless patients were interested in fertility preservation. Four patients $(28.5 \%)$ selected sperm cryopreservation and 1 patient $(7.1 \%)$ selected hemi-testicular preservation, as he had developed erectile dysfunction following previous surgery for colon cancer. Married patients and those with children did not wish to preserve fertility. In conclusion, infertility following chemotherapy raises serious concerns for young male patients with high-grade bone and soft tissue tumors. Prior to initiating chemotherapy, the potential risks of chemotherapy should be explained, counseling should be provided and informed consent should be obtained from the patients, ideally without delaying commencement of cancer treatment. Prior to administering cancer chemotherapy, orthopedic oncologists are encouraged to offer sperm banking to young male patients at risk of infertility.
\end{abstract}

Correspondence to: Dr Manabu Hoshi, Department of Orthopedic Surgery, Osaka City University Graduate School of Medicine, 1-4-3 Asahimachi, Abeno-ku, Osaka 545-8585, Japan

E-mail: hoshi@med.osaka-cu.ac.jp

Key words: fertility preservation, sperm cryopreservation, chemotherapy, high-grade malignant bone and soft tissue tumor

\section{Introduction}

Due to the advances in multidisciplinary therapy, including neoadjuvant chemotherapy (1), radiation therapy (2) and limb salvage surgery (3), the prognosis of high-grade bone and soft tissue tumors has improved markedly over the last few years and the number of long-term survivors has increased. Certain types of high-grade bone and soft tissue tumors, such as osteosarcoma, Ewing's sarcoma and synovial sarcoma, are most commonly encountered among adolescents and young adults. With the increase in the number of long-term survivors, latent treatment-related adverse effects have generated new concerns (4).

Post-chemotherapy infertility may be a major concern for young male cancer survivors (5). Established and safe techniques of sperm cryopreservation are currently available for such patients (6). Due to the recent developments in assisted reproductive technology, cryopreserved sperm is able to achieve fertilization (7). However, particularly in Japan, only few orthopedic oncologists recommend these measures against infertility to patients with high-grade bone and soft tissue tumors. To the best of our knowledge, there is currently only 1 available previous Japanese study on this topic, reporting that post-chemotherapy infertility is more common in male compared to female patients (8). We recently started to offer fertility preservation to our patients prior to cancer treatment. The purpose of this study was to present our findings regarding fertility preservation in Japanese male patients with malignant bone and soft tissue tumors.

\section{Materials and methods}

Patients. This prospective study included a total of 14 male patients, aged $<45$ years at diagnosis, who were treated for high-grade malignant bone and soft tissue tumors at the Osaka City University Hospital (Osaka, Japan) between January, 2008 and December, 2013. The median age at diagnosis was $23.0 \pm 12.0$ years (range, $8-42$ years).

Diagnostic procedure and staging. If such malignancies were suspected on the basis of physical examination and radiological findings, including radiograph, magnetic resonance imaging and computed tomography (CT), a needle or open biopsy was 
performed to obtain a histological diagnosis. All the biopsy specimens were examined by a pathologist specialized in sarcoma pathology and diagnosed according to the standard criteria for bone and soft tissue sarcoma subtyping (9). All the soft tissue sarcomas were graded according to the three-tier French system (10), with grade 2 and 3 tumors considered to be high-grade. The grading system for bone tumors (except Ewing's sarcoma) was based on that described by Broders (11), with grade 3 and 4 tumors considered to be high-grade. The clinical stage of each patient was evaluated according to the guidelines of the American Joint Committee on Cancer (AJCC) for soft tissue tumor and bone cancer $(12,13)$ following examination by lung CT and 2-deoxy-2- ${ }^{18} \mathrm{~F}$-fluoro-D-glucose positron emission tomography combined with $\mathrm{CT}$.

Treatment. All the patients with histologically proven high-grade bone tumors or soft tissue sarcomas (except for 1 patient with Ewing's sarcoma) were treated with pre- and postoperative caffeine-potentiated chemotherapy $(14,15)$ and underwent surgical resection of their tumors with wide resection margins (3). One patient with Ewing's sarcoma of the femur received high-dose intensive chemotherapy according to a modified form of the European Ewing Tumor Working Initiative of National Groups 1999 protocol (16) prior to tumor resection.

Fertility preservation planning. Regarding the timing of fertility preservation, once the biopsy material had been processed and the probability of high-grade malignant bone or soft tissue tumor raised, the provisional diagnosis, together with the treatment plan for surgery and chemotherapy and the expected prognosis, was explained to the patients (and their guardians, where appropriate) by orthopedic oncologists. The various potential treatment-related adverse effects, including the possibility of infertility after chemotherapy, were also explained. If the patient and their guardians were interested in fertility preservation, oncology-certified nurse specialists also participated in this discussion. Such patients were referred to a nearby reproductive specialist clinic for fertility preservation. In the majority of the cases, it was possible to initiate preoperative chemotherapy within 2 weeks of the initial visit.

The clinical characteristics of the patients, including age, gender, histopathology, type of tumor (soft tissue or bone), clinical stage, marital status and fatherhood status (childless or with children), together with the techniques of fertility preservation performed by the reproductive specialists, were documented.

This study was approved by the Ethics Committee of the Osaka City University Graduate School of Medicine and written informed consent was obtained from the patients or their guardians.

\section{Results}

Patient characteristics and fertility preservation. The relevant clinical characteristics of the patients and techniques for fertility preservation are summarized in Table I. A total of 7 patients presented with high-grade soft tissue tumors and the remaining 7 patients with high-grade bone tumors. The most common histopathological diagnosis was osteosarcoma
(6 patients). According to the AJCC clinical staging guidelines for soft tissue sarcomas, 3 cases had stage II, 3 had stage III and 1 had stage IV disease. According to the AJCC clinical staging guidelines for bone tumors, 1 case had stage IIA, 5 had stage IIB and 1 had stage IV disease. Of the 14 patients, 11 were unmarried and 3 were married. All 3 married patients already had children. Regarding the techniques for fertility preservation, 4 patients used sperm cryopreservation. One patient (case 5) had previously undergone surgery for colon cancer and had developed erectile dysfunction after surgery. That patient decided to undergo surgery for testicular cryopreservation under general anesthesia. Except for 1 patient (case 14), patients aged $<20$ years did not choose to preserve their sperm.

\section{Discussion}

The American Society of Clinical Oncology (17) proposed guidelines on fertility preservation for cancer patients in 2006 , which were updated in 2012 (18). The guidelines recommend that discussing the possibility of infertility with male patients who are to undergo cancer treatment during their reproductive years should be standard practice and healthcare providers must be prepared to refer all interested patients to appropriate reproductive specialists for consideration of sperm cryopreservation. However, in Japan, the majority of patients with high-grade malignant bone and soft tissue tumors are treated by orthopedic oncologists rather than chemotherapy specialists. These orthopedic oncologists usually explain various issues regarding chemotherapy protocols, the approach to tumor removal and the prognosis to patients with high-grade bone and soft tissue tumors prior to treatment initiation. However, they place little emphasis on chemotherapy-related adverse effects, such as infertility, and do not inform their patients of the availability of sperm cryopreservation. As the majority of the patients are primarily focused on cancer diagnosis and treatment, any concerns regarding possible future infertility tend to be secondary. However, similar to healthy individuals, the majority of young survivors would hope to be able to subsequently father children (19) and infertility may prove to be of great concern after marriage. The limited availability of published reports regarding fertility preservation in male patients with high-grade malignant bone and soft tissue tumors in Japan prompted us to undertake this prospective study.

Early treatment may contribute to the control of tumor growth and prevention of metastasis; therefore, no orthopedic oncologist should delay the initiation of cancer treatment. In this study, we explained the possibility of chemotherapy-related infertility to the patients immediately after the rapidly processed biopsy specimens had raised the possibility of a pathological diagnosis of high-grade malignancy and immediately referred interested patients to reproductive specialists for fertility preservation. During this short time, oncology-certified nurse specialists cooperated with the orthopedic oncologists to adequately inform the patients and/or their guardians on this topic. We also expedited the consultations between the reproductive specialists and interested patients. Once the final pathology report on the biopsy material had confirmed high-grade malignancy, we were able to initiate preoperative chemotherapy without delay. 
Table I. Relevant clinical and sociological patient characteristics and techniques of fertility preservation.

\begin{tabular}{|c|c|c|c|c|c|c|c|c|c|}
\hline Case & $\begin{array}{l}\text { Age, years } \\
\text { /gender }\end{array}$ & Histopathology & Type & $\begin{array}{c}\text { Anatomical } \\
\text { site }\end{array}$ & AJCC & Stage & $\begin{array}{l}\text { Marital } \\
\text { status }\end{array}$ & Offspring & $\begin{array}{c}\text { Fertility } \\
\text { preservation }\end{array}$ \\
\hline 1 & $16 / \mathrm{M}$ & Rhabdomyosarcoma & ST & Buttock & T2bN0M1 & IV & Unmarried & No & No \\
\hline 2 & $34 / \mathrm{M}$ & Synovial sarcoma & ST & Forearm & T1bN0M0 & II & Married & Yes & No \\
\hline 3 & 39/M & Synovial sarcoma & ST & Foot & T1aN0M0 & II & Married & Yes & No \\
\hline 4 & $34 / \mathrm{M}$ & $\begin{array}{l}\text { Extraskeletal } \\
\text { mesenchymal } \\
\text { chondrosarcoma }\end{array}$ & ST & Lower leg & T2bNOM0 & III & Married & Yes & No \\
\hline 5 & $37 / \mathrm{M}$ & $\begin{array}{l}\text { Etraskeletal myxoid } \\
\text { chondrosarcoma }\end{array}$ & ST & Thigh & T2bN0M0 & III & Unmarried & No & $\begin{array}{c}\text { Testicular } \\
\text { preservation }\end{array}$ \\
\hline 6 & $42 / \mathrm{M}$ & $\begin{array}{l}\text { Epithelioid } \\
\text { sarcoma }\end{array}$ & ST & Index finger & T1aN0M0 & II & Unmarried & No & $\begin{array}{c}\text { Sperm } \\
\text { preservation }\end{array}$ \\
\hline 7 & 28/M & $\begin{array}{l}\text { Extraskeletal } \\
\text { mesenchymal } \\
\text { chondrosarcoma }\end{array}$ & ST & Neck & T2bN0M0 & III & Unmarried & No & $\begin{array}{c}\text { Sperm } \\
\text { preservation }\end{array}$ \\
\hline 8 & $11 / \mathrm{M}$ & Ewing's sarcoma & Bone & Femur & T2N0M0 & IIB & Unmarried & No & No \\
\hline 9 & $13 / \mathrm{M}$ & Osteosarcoma & Bone & Femur & T2N0M0 & IIB & Unmarried & No & No \\
\hline 10 & $15 / \mathrm{M}$ & Osteosarcoma & Bone & Tibia & T2N0M0 & IIB & Unmarried & No & No \\
\hline 11 & $8 / \mathrm{M}$ & Osteosarcoma & Bone & Femur & T2N0M0 & IIB & Unmarried & No & No \\
\hline 12 & 27/M & Osteosarcoma & Bone & Humerus & T2N0M0 & IIB & Unmarried & No & $\begin{array}{c}\text { Sperm } \\
\text { preservation }\end{array}$ \\
\hline 13 & $9 / \mathrm{M}$ & Osteosarcoma & Bone & Femur & T2N0M1 & IV & Unmarried & No & No \\
\hline 14 & 19/M & Osteosarcoma & Bone & Humerus & T1N0M0 & IIA & Unmarried & No & $\begin{array}{c}\text { Sperm } \\
\text { preservation }\end{array}$ \\
\hline
\end{tabular}

AJCC, American Joint Committee on Cancer; M, male; ST, soft tissue.

Of the chemotherapeutic drugs, alkylating agents are reportedly the most toxic to the gonads (20). Of these, ifosfamide is currently considered a key drug for the treatment of high-grade bone and soft tissue tumors. Longhi et al (4) reported that 20/26 male patients had developed oligospermia or azoospermia following chemotherapy with high-dose ifosfamide (median dose, $42 \mathrm{~g} / \mathrm{m}^{2}$ ) for osteosarcoma. Williams et al (21) reported that gonadal dysfunction occurs at a total ifosfamide dose of $>60 \mathrm{~g} / \mathrm{m}^{2}$. Our standard chemotherapy protocol is a caffeine-assisted combination that contains high doses of ifosfamide $(14,15)$, the cumulative dose of this drug being $>45 \mathrm{~g} / \mathrm{m}^{2}$ for bone tumors and $>72 \mathrm{~g} / \mathrm{m}^{2}$ for soft tissue tumors. We consider infertility to be inevitable in male patients who have received this protocol.

Sperm cryopreservation, an easy and reliable means of fertility preservation in male patients, provides the possibility of future successful insemination with an assisted reproductive technique (22). Sperm cryopreservation is also emotionally beneficial for patients. Saito et al (23) reported that sperm cryopreservation improved the psychological status of young cancer patients during and after cancer treatment. In our study, unmarried and childless patients aged $>20$ years expressed a positive attitude towards fertility preservation and did preserve their sperm prior to commencing cancer treatment, whereas patients who already had children did not wish to preserve their sperm, stating that they did not wish for more children.

Lack of information regarding chemotherapy-related male infertility may generate serious future problems for cancer survivors. The most common reason for not banking sperm is lack of information regarding this option at the time of treatment planning. Only $60 \%$ of men reportedly recall being informed that infertility is an adverse effect of cancer treatment; however, oncologists suggest that patients may have forgotten information that was in fact provided to them (19). Certain doctors are not comfortable with this topic, stating reasons including lack of time in busy clinics and lack of knowledge regarding convenient sperm banking facilities (24). As the majority of Japanese orthopedic oncologists are generally not familiar or up-to-date with the topic of fertility, they fail to routinely provide male patients with adequate information on potential treatment-related infertility and the available options for preserving the ability to father children in the future.

The present study had several limitations. First, it was a small series and all the patients attended a single institution in Japan. Second, the provisional pathological diagnosis on biopsy specimens occasionally differs from the final diagnosis. Thus, providing comprehensive information based on the provisional diagnosis may lead to an unnecessary financial burden and mental anguish. We recommend explaining that this is a possibility. Finally, patients aged $\geq 20$ years are legally considered adults in Japan. We recommend that, prior to engaging in discussions with patients who are legally considered to be children (aged $<20$ years), orthopedic oncologists should seek approval from their parents or guardians regarding what information to provide to their offspring. Such information should include disclosing that the diagnosis is cancer. In 
accordance with these recommendations, we first discussed the topic of fertility preservation with the parents or guardians of such patients. Certain parents were uncomfortable with our suggestion that their sons receive information regarding fertility loss after chemotherapy and refused to grant permission. Therefore, some of the outcomes in this study were affected by the judgement of the patients' guardians.

In conclusion, sperm banking is clearly an effective way of preserving male fertility. To minimize delays in cancer treatment initiation, immediately after obtaining a provisional pathological diagnosis of high-grade bone or soft tissue sarcoma, orthopedic oncologists should obtain informed consent for treatment after providing information and counseling regarding the potential risks of chemotherapy. Orthopedic oncologists are encouraged to suggest sperm banking to young male patients at risk of infertility prior to initiating cancer chemotherapy.

\section{Acknowledgements}

We would like to thank Dr Kenichi Wakasa (Osaka City University Graduate School of Medicine, Osaka, Japan) for the histopathological diagnoses.

\section{References}

1. Meyers PA, Gorlick R, Heller G, et al: Intensification of preoperative chemotherapy for osteogenic sarcoma: results of the Memorial Sloan-Kettering (T12) protocol. J Clin Oncol 16 : 2452-2458, 1998.

2. Yang JC, Chang AE, Baker AR, et al: Randomized prospective study of the benefit of adjuvant radiation therapy in the treatment of soft tissue sarcomas of the extremity. J Clin Oncol 16: 197-203, 1998.

3. Kawaguchi N, Ahmed AR, Matsumoto S, Manabe J and Matsushita Y: The concept of curative margin in surgery for bone and soft tissue sarcoma. Clin Orthop Relat Res 419: 165-172, 2004.

4. Longhi A, Ferrari S, Tamburini A, et al: Late effects of chemotherapy and radiotherapy in osteosarcoma and Ewing sarcoma patients: the Italian Sarcoma Group Experience (1983-2006). Cancer 118: 5050-5059, 2012.

5. Schover LR, Brey K, Lichtin A, Lipshultz LI and Jeha S: Knowledge and experience regarding cancer, infertility, and sperm banking in younger male survivors. J Clin Oncol 20: 1880-1889, 2002.

6. Kelleher S, Wishart SM, Liu PY, et al: Long-term outcomes of elective human sperm cryostorage. Hum Reprod 16: 2632-2639, 2001.

7. Palermo GD, Cohen J, Alikani M, Adler A and Rosenwaks Z: Intracytoplasmic sperm injection: a novel treatment for all forms of male factor infertility. Fertil Steril 63: 1231-1240, 1995.
8. Yonemoto T, Ishii T, Takeuchi Y, Hagiwara Y, Iwata S and Tatezaki S: Recently intensified chemotherapy for high-grade osteosarcoma may affect fertility in long-term male survivors. Anticancer Res 29: 763-767, 2009.

9. Flecher CDM, Bridge JA, Hogendoorn PCW and Mertens F (eds): WHO Classification of Tumors of Soft Tissue and Bone. 4th edition. IARC Press, Lyon, 2002.

10. Trojani M, Contesso G, Coindre JM, et al: Soft-tissue sarcomas of adults; study of pathological prognostic variables and definition of a histopathological grading system. Int J Cancer 33: 37-42, 1984.

11. Broders AC: Squamous cell epithelioma of the lip: a study of 537 cases. JAMA 74: 656-664, 1920.

12. Edge SB, Byrd DR, Compton CC, et al (eds): Soft tissue sarcoma. In: AJCC Cancer Staging Manual. 7th edition. Springer, New York, NY, pp291-296, 2010.

13. Edge SB, Byrd DR, Compton CC, et al (eds): Bone. In: AJCC Cancer Staging Manual. 7th edition. Springer, New York, NY, pp281-290, 2010.

14. Tsuchiya H, Tomita K, Mori Y, Asada N and Yamamoto N: Marginal excision for osteosarcoma with caffeine assisted chemotherapy. Clin Orthop Relat Res 358: 27-35, 1999.

15. Takeuchi A, Tsuchiya H, Yamamoto N, et al: Caffeine-potentiated chemotherapy for patients with high-grade soft tissue sarcoma: long-term clinical outcome. Anticancer Res 27: 3489-3495, 2007.

16. Juergens C, Weston C, Lewis I, et al: Safety assessment of intensive induction with vincristine, ifosfamide, doxorubicin, and etoposide (VIDE) in the treatment of Ewing tumors in the EURO-E.W.I.N.G. 99 clinical trial. Pediatr Blood Cancer 47: 22-29, 2006.

17. Lee SJ, Schover LR, Partridge AH, et al; American Society of Clinical Oncology: American Society of Clinical Oncology recommendations on fertility preservation in cancer patients. J Clin Oncol 24: 2917-2931, 2006.

18. Loren AW, Mangu PB, Beck LN, et al; American Society of Clinical Oncology: Fertility preservation for patients with cancer: American Society of Clinical Oncology clinical practice guideline update. J Clin Oncol 31: 2500-2510, 2013.

19. Schover LR, Rybicki LA, Martin BA and Bringelsen KA: Having children after cancer. A pilot survey of survivors' attitudes and experiences. Cancer 86: 697-709, 1999.

20. Kenney LB, Laufer MR, Grant FD, Grier H and Diller L: High risk of infertility and long term gonadal damage in males treated with high dose cyclophosphamide for sarcoma during childhood. Cancer 91: 613-621, 2001.

21. Williams D, Crofton PM and Levitt G: Does ifosfamide affect gonadal function? Pediatr Blood Cancer 50: 347-351, 2008.

22. Lass A, Akagbosu F, Abusheikha N, et al: A programme of semen cryopreservation for patients with malignant disease in a tertiary infertility centre: lessons from 8 years' experience. Hum Reprod 13: 3256-3261, 1998.

23. Saito K, Suzuki K, Iwasaki A, Yumura Y and Kubota Y: Sperm cryopreservation before cancer chemotherapy helps in the emotional battle against cancer. Cancer 104: 521-524, 2005.

24. Schover LR, Brey K, Lichtin A, Lipshultz LI and Jeha S: Oncologists' attitudes and practices regarding banking sperm before cancer treatment. J Clin Oncol 20: 1890-1897, 2002. 